\title{
The Ancestral Hemochromatosis Haplotype Is Associated With a Severe Phenotype Expression in Italian Patients
}

\author{
Alberto Piperno, ${ }^{1}$ Cristina Arosio, ${ }^{2}$ Silvia Fargion, ${ }^{3}$ Antonella Roetto,${ }^{4}$ Carlo Nicoli, ${ }^{1}$ Domenico Girelli, ${ }^{5}$ \\ Luca Sbaiz, ${ }^{4}$ Paolo Gasparini, ${ }^{6}$ Giuseppe Boari, ${ }^{8}$ Maurizio Sampietro, ${ }^{3}$ And Clara Camaschella ${ }^{4,7}$
}

\begin{abstract}
We evaluate the relation between genotype and phenotype in 47 Italian male patients with homozygous genetic hemochromatosis (GH). Phenotype evaluation was based on the ratio of amount of iron removed (IR) by phlebotomy and age (IR/age). Patients were divided in two classes of phenotype expression: class I included 26 patients with less severe iron overload (IR/age $\leq \mathbf{0 . 3 3})$ and class II included 21 patients with a more marked one (IR/age $>0.33)$. Genetic variability was assessed by haplotype analysis combining alleles at HLA-B, D6S265, HLA-A, and D6S105 loci. A common ancestral haplotype carrying D6S265-1, HLA-A3, and D6S105-8 alleles was present in 13 of $52(25 \%)$ chromosomes in class I and in 24 of $42(57 \%)$ chromosomes in class II $(P=.0027)$. Homozygotes and heterozygotes for the ancestral haplotype had higher iron indices than patients carrying two haplotypes other than the ancestral one. Seven of the eight patients homozygous for the ancestral haplotype were in class II, heterozygotes were equally distributed between the two classes, whereas 14 of 18 carriers of other haplotype combinations were in class I. Our results suggest that the gene defect linked to the ancestral haplotype is the result of a single, severe mutation. The high variability of phenotype expression in heterozygotes for the ancestral haplotype could be accounted for the contribution of the mutation carried by the second haplotype. Combination of different mutations could be responsible for the variable degrees of iron overload found in patients with GH. (HePATOLOGY 1996;24:43-46.)
\end{abstract}

Both clinical presentation and body iron stores differ in patients with genetic hemochromatosis (GH). Women usually develop symptoms and signs of the disease later in their life because of menstrual blood losses and pregnancy. Age, dietary habits, and factors such as blood donations and blood losses can modify hepatic iron stores. Heavy alcohol intake and chronic viral hepatitis facilitate the development of liver damage in patients with GH. ${ }^{1,2}$ Phenotypic concordance between siblings with homozygous GH provides evidence that

Abbreviations: GH, genetic hemochromatosis; HII, hepatic iron index; IR, iron removed TS, transferrin saturation; SF, serum ferritin; LIC, liver iron concentration.

From the ${ }^{1}$ Istituto di Scienze Biomediche, Divisione di Medicina 1, Ospedale S. Gerardo, Monza, Italy; ${ }^{2}$ Centro Auxologico Italiano, Milan, Italy; ${ }^{3}$ Istituto di Medicina Interna e Fisiopatologia Medica, Università di Milano, Milan, Italy; ${ }^{4}$ Dipartimento di Scienze Biomediche e Oncologia Umana, Universita di Torino, Torino, Italy; ${ }^{5}$ Istituto di Clinica Medica, Università di Verona, Verona, Italy; ${ }^{6}$ I.R.C.C.S., Ospedale C.S.S. San Giovanni Rotondo (Foggia), Italy; ${ }^{7} \mathrm{CNR}$ - CIOS - Torino, Italy; and ${ }^{8}$ Istituto di Statistica, Università Cattolica S. Cuore, Milano, Italy.

Received June 14, 1995; accepted February 21, 1996.

Partially supported by Telethon, Rome, Italy, grant 429; and Ministero dell'Università per la Ricerca Scientifica e Tecnologica (MURST), Rome, Italy to Dr. Camaschella.

Address reprint requests to: Dr. A. Piperno, Divisione di Medicina I, Ospedale S. Gerardo, Via Donizetti 106, 20052-Monza, Italy.

Copyright (C) 1996 by the American Association for the Study of Liver Diseases. 0270-9139/96/2401-0008\$3.00/0 the expression of the disease is affected by genetic factors. ${ }^{3,4}$ Haplotype analysis in Australian patients of Irish or Scottish origin provides evidence of a common ancestral haplotype characterized by the combination of D6S265-1, HLA-A3, D6S105-8 alleles. ${ }^{5}$ Similarly, this haplotype accounts for about $30 \%$ of the chromosomes carrying the $\mathrm{GH}$ gene in Italy ${ }^{6}$ and up to $50 \%$ of GH chromosomes in England and France ${ }^{7,8}$ whereas it is very infrequent in those with normal levels. Very recently, Crawford et al. ${ }^{9}$ showed that Australian GH patients with two copies of the ancestral haplotype have significantly higher hepatic iron index (HII) than those carrying only one copy or none, suggesting that ancestral haplotype is associated with a common mutation. Besides the HLAA3 linked haplotype, a further haplotype restricted to $\mathrm{GH}$ chromosomes, which possesses HLA-A11 and the allele 2 of HLA-F (restriction fragment length polymorphism), has been described. ${ }^{10}$ It is likely that this haplotype harbors a second independent mutation different from the ancestral one. Several other haplotypes have been found in $\mathrm{GH}^{5,6,8}$ suggesting the possibility of heterogeneous molecular defects in contrast to the original hypothesis of a unique ancestral mutation as responsible for all GH cases in white population. ${ }^{11}$ Multiple mutations are commonly found in other genetic disorders and may account for different severity of those diseases.

In this study we evaluate whether genetic heterogeneity as defined by haplotype analysis could account for phenotype heterogeneity in a group of 47 Italian patients with homozygous $\mathrm{GH}$.

\section{PATIENTS AND METHODS}

Subjects. We studied 47 unrelated men with homozygous GH from three different clinical centers in northern Italy. They were aged 25 to 68 years (mean $\pm \mathrm{SD}: 44.5 \pm 11$ ). Inclusion criteria were: (1) no known causes of secondary iron overload; (2) hepatocellular hemosiderin deposits of $\mathrm{III}^{\circ}-\mathrm{IV}^{\circ}$; (3) HII greater than 2 and/or a total amount of iron removed (IR) by phlebotomy to achieve iron depletion higher than $5 \mathrm{~g}$; and (4) availability of relatives to perform molecular studies and unambiguously assign haplotypes linked to the GH gene. Exclusion criteria were: (1) female sex; (2) history of chronic blood losses or blood donations; and (3) history of blood transfusions or parenteral iron administration.

The presence of fibrosis or cirrhosis was determined at liver biopsy. Cardiopathy was based on physical signs of heart failure and/or left ventricular dilatation with low left ventricular ejection fraction and fractional shortening at echocardiography, or arrythmias requiring medical therapy. Patients were considered to be diabetic in the presence of hyperglycemia requiring oral hypoglycemic agents or insulin; the presence of insulin-dependent diabetes was confirmed by low Cpeptide levels at fasting and 6 minutes after intravenous glucagon (1 mg) infusion. Patients who complained of impotence or decreased libido with low serum testosterone, follicle stimulating hormone and luteinizing hormone levels were considered affected by pituitary hypogonadism. Presence of arthritis was based on history, clinical symptoms, and physical examination.

Iron Status. Transferrin saturation (TS) and serum ferritin (SF) concentration were measured in all patients by standard methods. Liver iron concentration (LIC) was determined by atomic absorption spectrophotometry (Perkin-Elmer S2380, Norwalk, CT) in 42 pa- 


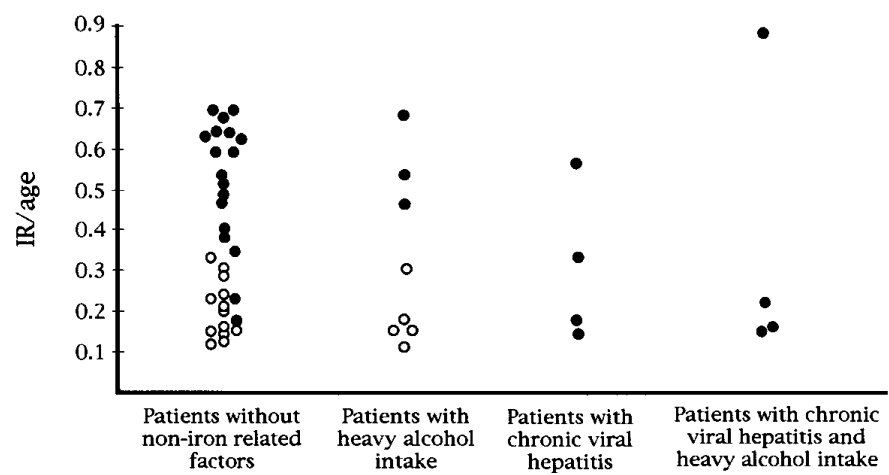

FIG. 1. Distibution of the ratio of total IR by phlebotomy and age (IR/age) in 47 patients with genetic hemochromatosis divided according to the presence of liver fibrosis and non-iron-related factors (heavy alcohol intake [ $\geq 80 \mathrm{~g} / \mathrm{d}$ ] and chronic viral hepatitis or both). Patients with liver fibrosis: 9 ; Patients without liver fibrosis: $\bigcirc$.

tients, in fresh $(\mathrm{N}=24)$ or deparaffinized $(\mathrm{N}=18)$ specimens. Paraffin-embedded samples, weighing more than $0.5 \mathrm{mg}$, were prepared as described by Olynik et al. ${ }^{12}$ IR was determined in all the patients on the basis of the amount of blood removed by weekly phlebotomies $(400 \mathrm{~mL})$ until the achievement of iron depletion $(1 \mathrm{~mL}$ of blood removed corresponding to $0.5 \mathrm{mg}$ of iron). To correct for the agedependency of iron overload in GH, HII (ratio of LIC $[\mu \mathrm{mol} / \mathrm{g}]$ and age [years]) and the ratio between IR (g) and age (years) (IR/age) were calculated. The achievement of iron depletion was defined by an SF lower than $30 \mu \mathrm{g} / \mathrm{L}$ in the presence of a TS less than $30 \%$ and a mild anemia that did not promptly recover after cessation of phlebotomies.

Phenotype Evaluation. Evaluation was based on clinical manifestations of GH and on the amount of body iron overload. IR was considered as the most reliable estimate of total iron burden because of the observed saturability of liver iron deposits with an increasing amount of total iron stores. In fact, to understand the relationship between LIC and IR, the following two models were compared: the simple linear (LIC $=\mathrm{h}+\mathrm{k} \cdot \mathrm{IR}$ ) and the straight-line reciprocal (LIC $=\mathrm{IR} /[\mathrm{a}+\mathrm{b} \cdot \mathrm{IR}])$, that shows an asymptotical behavior. The best significant linear relation was achieved by a model with null intercept $(h=0)$ which gave an $\mathrm{R}^{2}=0.54$; nevertheless, the nonlinear model showed a better fit to data, with $\mathrm{R}^{2}=0.76$. Both fitted models were highly significant $(P<.01)$ and passed through the origin. Finally, to divide patients in two groups of phenotype severity, the attention was focused on IR/age values, because of the age-dependency of iron overload in GH. ${ }^{1}$ To define the cutoff level we first plotted the IR/age values of patients according to the presence/absence of liver fibrosis. Moreover, because the development of liver fibrosis in GH is related to the amount of iron overload, but is also highly influenced by the presence of non-iron-related factors ${ }^{1,2}$ alcohol intake and chronic hepatitis infection were also considered in this calculation. As shown in Fig. 1, patients with associated chronic hepatitis, with or without heavy alcohol intake, had liver fibrosis for low as well as high IR/age values, so they were not used for defining the cutoff. In all the other patients the cutoff level that maximizes the specificity of a severe phenotype in $\mathrm{GH}$ (100\% of patients with liver fibrosis) is 0.33 . The same conclusion can be drawn in the same patients considering a parametric approach. In fact, in patients without liver fibrosis, the IR/age has a mean value $(\mathrm{m})=0.198$ and a standard deviation $(\sigma)=0.07$, and in patients with liver fibrosis $\mathrm{m}=0.52$ and $\sigma=0.15$. Assuming a gaussian distribution for the corresponding parent populations, it can be verified that the previously established cutoff point of 0.33 corresponds to definitively satisfying levels of specificity (0.96) and sensitivity (0.90). Thus, we define a less severe class of phenotype expression (class I) including patients with an IR/age of less than or equal to 0.33 and a more severe one (class II) including patients with an IR/age greater than 0.33 .

Genotype Evaluation. Forty-seven families consisting of a total number of 195 individuals were studied. Genetic variability in probands was assessed by analyzing HLA class I antigens (A and B) and two other markers (D6S265 and D6S105) that show strong linkage disequilibrium with GH in Italian patients. ${ }^{6}$ HLA-A and -B antigens were typed by microlymphocytotoxicity test; polymorphic alleles of the D6S265 and D6S105 microsatellites were analyzed by polyacrylamide gel electrophoresis after polymerase chain reaction amplification with specific primers. ${ }^{13,14}$ Allele sizes were defined using either a semiautomated fluorescence-based technology or using ethidium bromide staining and appropriate markers for size control. ${ }^{6,15}$ Haplotypes were determined on the basis of allele inheritance within families combining alleles at different loci in the following order: HLAB, D6S265, HLA-A, D6S105 according to physical mapping.,5,16

The full ancestral haplotype is characterized by the alleles 7,1 , 3,8 at the four polymorphic markers. Because the HLA-B7 allele is not an independent marker of the $\mathrm{GH}$ gene ${ }^{11}$ haplotypes carrying alleles 1, 3, 8 associated to HLA-B alleles other than 7 were also considered to be of ancestral origin. Moreover, because of the mechanism of slippage, which can generate new alleles at dinucleotide microsatellites, haplotypes differing from the ancestral one by 2 basepairs at a single marker were considered ancestral, as previously suggested. ${ }^{5}$ Thus, we define as the ancestral haplotype that characterized by the following alleles: HLA-Bx, D6S265-1, HLA-A3, D6S105-8/7/9. The same rule was applied for D6S105 alleles related to other haplotypes.

Statistical Analysis. The nonparametric Mann-Whitney $U$ test and the Fisher exact $U$ test were used to evaluate differences between groups of patients. They were calculated with the statistical package InStat 2.01 (GraphPad Software, San Diego, CA, 1993). The ordinary least squares method was used to investigate the relation between LIC and IR and the reciprocal transformation was performed to achieve linearity in the straight-line reciprocal model. The determination coefficient $R^{2}$ was calculated to evaluate the model's goodness of fit.

\section{RESULTS}

Table 1 reports the main biochemical and clinical characteristics of the patients divided in the two classes of phenotype severity. Class I included 26 patients and class II included 21 patients. Class II patients had higher TS, SF, LIC, HII, IR and, IR/age values than class I patients. Furthermore, liver fibrosis and cirrhosis, insulin-dependent diabetes, heart failure, and pituitary hypogonadism were significantly more frequent in class II than in class I patients.

TABle 1. Main Clinical and Biochemical Data of Patients With GH

\begin{tabular}{lccc}
\hline & $\begin{array}{c}\text { Class I } \\
(\mathbf{n}=\mathbf{2 6})\end{array}$ & $\begin{array}{c}\text { Class II } \\
(\mathbf{n}=\mathbf{2 1})\end{array}$ & $\boldsymbol{P}$ \\
\hline Age (yr) & $46 \pm 13$ & $43 \pm 9$ & $\mathrm{NS}$ \\
& $(25-68)$ & $(25-59)$ & \\
Liver fibrosis or cirrhosis & $8(31 \%)$ & $21(100 \%)$ & $<.0001$ \\
IDDM & $1(4 \%)$ & $7(33 \%)$ & $=.015$ \\
NIDDM & $4(15 \%)$ & - & $\mathrm{NS}$ \\
Cardiopathy & $1(4 \%)$ & $6(28 \%)$ & $=.035$ \\
Hypogonadism & $1(4 \%)$ & $9(43 \%)$ & $<.005$ \\
Arthropathy & $1(4 \%)$ & $2(9.5 \%)$ & $\mathrm{NS}$ \\
TS (\%) & $73 \pm 13$ & $86 \pm 10$ & $<.005$ \\
& $(56-98)$ & $(69-100)$ & \\
SF ( $\mu$ g/L) & $1440 \pm 907$ & $4319 \pm 2503$ & $<.0001$ \\
& $(600-4040)$ & $(725-10990)$ & \\
LIC ( $\mu$ g/100 mg) & $1310 \pm 487 *$ & $2622 \pm 506 \dagger$ & $<.0005$ \\
& $(637-2835)$ & $(1311-3556)$ & \\
HII & $5 \pm 1.9 *$ & $11.3 \pm 2.8 \dagger$ & $<.0001$ \\
& $(3.2-9.8)$ & $(7.6-18.6)$ & \\
IR (g) & $8.7 \pm 2.5$ & $24.2 \pm 6.3$ & $<.0001$ \\
IR/age & $(5-15)$ & $(10-37)$ & \\
Non-iron-related factors & $0.2 \pm 0.07$ & $0.58 \pm 0.12$ & $<.0001$ \\
& $(0.11-0.33)$ & $(0.34-0.88)$ & \\
& $11(42 \%)$ & $5(24 \%)$ & $\mathrm{NS}$ \\
\hline
\end{tabular}

NOTE. Patients divided in two classes of severity according to the ratio of total IR and age (IR/age). Class I includes patients with IR/age $\leq 0.33$. Class II includes patients with IR/age $>0.33$. Non-iron-related factors: heavy alcohol intake ( $\geq 80 \mathrm{~g} / \mathrm{d}$ ) and/or chronic viral hepatitis.

Abbreviations: IDDM: insulin-dependent diabetes mellitus; NIDDM: noninsulin-dependent diabetes mellitus.

* Performed in 22 patients.

$\dagger$ Performed in 20 patients. 
Table 2 shows the prevalence of the more common haplotypes in the two classes. Ancestral haplotype was present in 13 of $52(25 \%)$ chromosomes in class I patients as compared with 24 of $42(57 \%)$ in class II. HLA-B7 and HLA-B35 were the two most frequent HLA-B alleles associated with the ancestral haplotype. Another haplotype characterized by the presence of alleles D6S265-4, HLA-A11, and D6S105-7/6/8 was present in 5 of $52(10 \%)$ chromosomes in class I and was not found in class II patients. No other differences of haplotype distribution were observed between the two groups of patients.

Eight patients were homozygous for the ancestral haplotype, 21 were heterozygous, and 18 carried two different haplotypes not related to the ancestral one (carriers of other haplotype combinations). Table 3 shows the main clinical and biochemical data of patients divided according to haplotype combinations. Liver fibrosis or cirrhosis was more frequent in homozygotes and heterozygotes for the ancestral haplotype than in those carrying two different haplotypes. Homozygotes for the ancestral haplotype were younger than the other two groups of patients but the difference was not statistically significant. They had significantly higher HII, IR, and IR/ age values than those observed in carriers of other haplotype combinations. Heterozygotes for the ancestral haplotype had higher SF, IR, and IR/age than carriers of other haplotype combinations.

Figure 2 reported the number of patients subdivided according to haplotype combinations and phenotype expression. The majority of patients homozygotes for the ancestral haplotype (7 of 8) were in class II. Heterozygotes for the ancestral haplotype were equally distributed between the two groups (11 in class I and 10 in class II), whereas the majority of patients carrying two different haplotypes not related to the ancestral one (14 of 18) were in class I.

\section{DISCUSSION}

An ancestral haplotype, which probably carries the original mutation of $\mathrm{GH}$, is present in $30 \%$ to $50 \%$ of GH chromosomes in different populations. ${ }^{5-8}$ Our results suggest that the gene defect linked to the ancestral haplotype is the result of a single, severe mutation. In fact, in our series, patients homozygous for the ancestral haplotype have HII, IR, and IR/age values higher than in patients not carrying the ancestral haplotype. To further analyze the relationship between phenotype and genotype in GH we divided patients in two classes

Table 2. Most Frequent Haplotypes Deriving From Combination of Alleles at HLA-B, DS6265, HLA-A, D6S105 Loci in GH Patients

\begin{tabular}{lccc}
\hline \multicolumn{1}{c}{ Haplotype } & $\begin{array}{c}\text { Class I } \\
(\mathbf{N}=\mathbf{5 2})\end{array}$ & $\begin{array}{c}\text { Class II } \\
(\mathbf{N}=\mathbf{4 2})\end{array}$ & $\boldsymbol{P}$ \\
\hline $7,1,3,8 / 7 / 9$ & $4(0.08)$ & $10(0.24)$ & .041 \\
$5,1,3,8 / 7$ & - & $4(0.09)$ & \\
$12,1,3,8$ & $1(0.02)$ & $2(0.05)$ & \\
$27,1,3,8$ & $1(0.02)$ & $3(0.07)$ & \\
$35,1,3,8 / 9$ & $4(0.08)$ & $2(0.05)$ & \\
x, $1,3,8 / 9$ & $3(0.06)$ & $3(0.07)$ & \\
Ancestral haplotypes (all) & $13(0.25)$ & $24(0.57)$ & .0027 \\
x, 3, $1,8 / 9$ & $2(0.04)$ & $2(0.05)$ & NS \\
$35,3,3,5$ & $2(0.04)$ & - & NS \\
x, 3, 9, 6/5/7 & $5(0.1)$ & $4(0.09)$ & NS \\
x, $4,11,7 / 6 / 8$ & $5(0.1)$ & - & .063 \\
x, 5, 2, 6/7 & $3(0.06)$ & $2(0.05)$ & NS \\
x, 5, 2, 9/8 & $4(0.08)$ & $1(0.02)$ & NS \\
$5,6,2,5 / 6$ & $2(0.04)$ & $1(0.02)$ & NS \\
Other haplotypes & $16(0.31)$ & $8(0.19)$ & NS \\
\hline
\end{tabular}

NOTE. Patients divided in two classes of severity according to the ratio of total IR and age (IR/age). Class I includes 26 patients with IR/age $\leq 0.33$. Class II includes 21 patients with IR/age $>0.33$.

Abbreviation: NS, not significant.
TABle 3. Main Clinical and Biochemical Data of Patients Divided According to Haplotype Combinations

\begin{tabular}{lccc}
\hline & $\begin{array}{c}\text { Ancestral } \\
\text { Haplotype } \\
\text { Homozygotes } \\
(\mathbf{N}=\mathbf{8})\end{array}$ & $\begin{array}{c}\text { Ancestral } \\
\text { Haplotype } \\
\text { Heterozygotes } \\
(\mathbf{N}=\mathbf{2 1})\end{array}$ & $\begin{array}{c}\text { Other } \\
\text { Haplotype } \\
\text { Carriers } \\
(\mathbf{N}=\mathbf{1 8})\end{array}$ \\
\hline Age (yr) & $39 \pm 12$ & $45 \pm 9.7$ & $46.3 \pm 12$ \\
Liver fibrosis or cirrhosis & $7(87.5 \%) \dagger$ & $16(76 \%) \dagger$ & $6(33.3 \%)$ \\
IDDM & $2(25 \%)$ & $4(19 \%)$ & $2(11 \%)$ \\
NIDDM & - & $3(14 \%)$ & $1(5.6 \%)$ \\
Cardiopathy & $2(25 \%)$ & $3(14 \%)$ & $2(11 \%)$ \\
Hypogonadism & $2(25 \%)$ & $7(33 \%)$ & $1(5.5 \%)$ \\
Arthropathy & - & $2(9 \%)$ & $1(5.5 \%)$ \\
TS (\%) & $75 \pm 55.2$ & $81.2 \pm 13.7$ & $78.2 \pm 13.3$ \\
& $(69-80)$ & $(56-100)$ & $(57-100)$ \\
SF ( $\mu$ g/L) & $2882 \pm 1935$ & $3147 \pm 2329 \S$ & $2039 \pm 2328$ \\
& $(627-5800)$ & $(600-10990)$ & $(608-8000)$ \\
LIC ( $\mu$ g/100 mg)* & $2280 \pm 950$ & $2078 \pm 806$ & $1660 \pm 730$ \\
& $(637-3210)$ & $(845-3556)$ & $(804-3387)$ \\
HII* & $10.6 \pm 4.7 \dagger$ & $8.4 \pm 3.7$ & $6.6 \pm 3.3$ \\
& $(4.1-18.6)$ & $(3.2-14.4)$ & $(3.2-14.2)$ \\
IR (g) & $20 \pm 9.4 \|$ & $17.5 \pm 9.5 \|$ & $11 \pm 6.2$ \\
& $(6-32.1)$ & $(7-37)$ & $(5-24)$ \\
IR/age & $0.5 \pm 0.15 \uparrow$ & $0.41 \pm 0.23 \|$ & $0.25 \pm 0.16$ \\
& $(0.21-0.69)$ & $(0.14-0.88)$ & $(0.11-0.67)$ \\
Non-iron-related factors & $2(25 \%)$ & $9(42.8 \%)$ & $5(27.7 \%)$ \\
\hline
\end{tabular}

NOTE. Non-iron-related factors: heavy alcohol intake ( $\geq 80 \mathrm{~g} / \mathrm{d})$ and/or chronic viral hepatitis.

Abbreviations: IDDM: insulin-dependent diabetes mellitus; NIDDM: noninsulin-dependent diabetes.

* LIC and HII were measured in 7 ancestral haplotype homozygotes, in 18 heterozygotes, and in 17 carriers of other haplotype combinations.

$\dagger P<.04$.

$\ddagger P<.02$.

$\S P<.01$.

$\| P<.03$.

I $P<.005$ vs. carriers of other haplotypes.

of severity according to IR/age values. As extensively described in the Patients and Methods section, a cutoff level of 0.33 was chosen based on the presence or absence of liver fibrosis, excluding the effect of non-iron-related factors on the development of liver fibrosis. The marked severity of phenotype expression in class II patients is further shown by the higher prevalence of insulin-dependent diabetes, cardiopathy, and pituitary hypogonadism as compared with class I patients. The ancestral mutation was more frequent in class

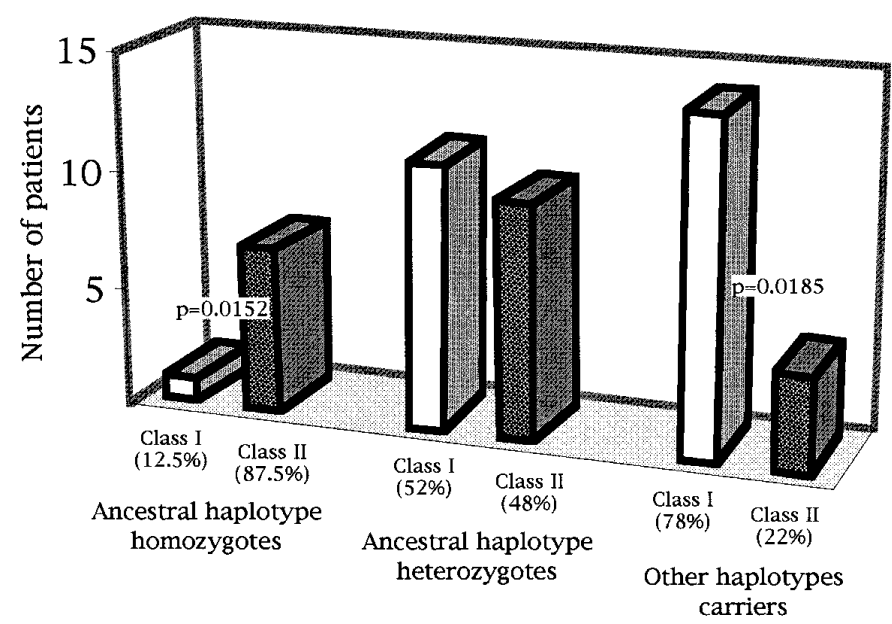

FIG. 2. Number of patients subdivided according to genotype and class of phenotype severity. Class I includes patients with a ratio of total IR by phlebotomy and age $($ IR/age $) \leq 0.33$. Class II includes patients with IR/age $>0.33$. 
II than in class I patients supporting the hypothesis of a severe mutation linked to the ancestral haplotype. According to this hypothesis, the majority $(87.5 \%)$ of homozygotes for the ancestral haplotype were recorded in class II.

In agreement with our data, Crawford et al. ${ }^{9}$ recently found higher HII values in patients homozygous for the ancestral haplotype compared with patients who did not carry the ancestral haplotype. However, in our series, we did not find significant differences between ancestral haplotype homozygotes and heterozygotes, whereas both had significantly higher IR and IR/age values and a higher prevalence of liver fibrosis than carriers of other haplotypes. The difference between the two studies may be partially related to the different evaluation of patients' phenotype, based on IR and its derived IR/age parameter in our study and on LIC and HII in the study by Crawford et al. In our data, the correlation between LIC and IR is not linear and LIC has a trend to a plateau as body iron overload increases, as previously shown by Mandelli et al. ${ }^{17}$ This indicates that IR is the best estimate of total iron burden and IR/age is the best index to evaluate the rate of body iron accumulation in GH. Looking at the distribution of IR/age values in patients heterozygous for the ancestral haplotype we observed that they were equally distributed between the two classes of phenotype severity (Fig. 2 ). Thus, the high variability of phenotype expression observed in this group of patients could be accounted for the contribution of the mutation carried by the second haplotype.

Among chromosomes carrying the ancestral haplotype, six were associated with the A3, B35 haplotype, all of them found in patients originating from northeastern Italy. This data confirm that A3, B35 haplotype in northeastern Italy is the result of an ancient recombination of the ancestral haplotype $^{18}$ and differs from that reported in an Australian population where HLA-A3 on a haplotype with B35 is never associated with the ancestral haplotype. ${ }^{5}$

Patients carrying two different haplotypes not related to the ancestral one generally have a less severe phenotype. In fact, two thirds of them were classified in class I. Among patients of class I, we found a second, more frequent haplotype carrying alleles D6S265-4 and HLA-A11 that was not found in class II patients. This haplotype might correspond to the second haplotype reported to be restricted to GH chromosomes in France, characterized by HLA-A11, HLA-F (restriction fragment length polymorphism) allele $2 .{ }^{10}$ Although our finding needs confirmation in a larger series, the mild phenotype associated with this haplotype is in agreement with the hypothesis of a second independent mutation. All the other haplotypes were equally distributed between the two classes of phenotype severity, and it was not possible to identify other haplotype combinations associated to homogeneous patterns of phenotype expression. The large heterogeneity of these haplotypes and their similar frequency in $\mathrm{GH}$ patients and in controls ${ }^{6}$ suggest that several GH mutations may exist in the Italian population. Combination of different mutations could be responsible for the variable degrees of iron overload found in $\mathrm{GH}$ patients. On this genetic background, age, sex, dietary habits, and other acquired factors act modelling the clinical presentation of homozygous $\mathrm{GH}$ patients.
Acknowledgment: We thank Eric Rappaport and Paolo Fortina for their technical support in the characterization of D6S105 alleles. We are indebted to Maristella Farè for HLA typing and Clara Sincich for technical assistance in preparing the manuscript.

\section{REFERENCES}

1. Bassett ML, Halliday JW, Powell LW. Value of hepatic iron measurements in early hemochromatosis and determination of the critical level associated with fibrosis. HePATOLOGY 1986;6:24-29.

2. Piperno A, Fargion S, D’Alba R, Roffi L, Fracanzani AL, Vecchi L, Failla M, et al. Liver damage in Italian patients with hereditary hemochromatosis is highly influenced by hepatitis B and C virus infection. J Hepatol 1992;16: 364-368

3. Muir AW, McLaren GD, Braun W, Askari A. Evidence for heterogeneity in hereditary hemochromatosis. Evaluation of 174 persons in nine families. Am J Med 1984; 76:806-814.

4. Crawford D, Halliday JW, Summers KM, Bourke MJ, Powell LW. Concordance of iron storage in siblings with genetic hemochromatosis: evidence for a predominantly genetic effect on iron storage. HEPATOLOGY 1993; 17 : 833-837.

5. Jazwinska EC, Pyper W, Burt M, Francis J, Goldwurm S, Webb S, Lee S, et al. Haplotype analysis in Australian hemochromatosis patients: evidence for a predominant ancestral haplotype exclusively associated with hemochromatosis. Am J Hum Genet 1995;56:428-433.

6. Camaschella C, Roetto A, Gasparini P, Piperno A, Fortina R, Surrey S, Rappaport E. Allelic association of microsatellites of $6 \mathrm{p}$ in Italian hemochromatosis patients. Hum Genet 1996; 97:476-481.

7. Raha-Chowdury R, Bowen D, Stone C, Pointon J, Terwilliger J, Shearman J, Robson K, et al. New polymorphic microsatellite markers place the hemochromatosis gene telomerically to D6S105. Hum Mol Genet 1995; 4: 1869-1874.

8. Worwood M, Gasparini P, Camaschella C. Report on the International Workshop on Molecular Genetics of Hemochromatosis held at Villa Feltrinelli Gargnano (BS), Italy, 25th September 1994. J Med Genet 1995;32: 320-327.

9. Crawford D, Powell L, Leggett B, Francis J, Fletcher L, Webb S, Halliday $\mathrm{J}$, et al. Evidence that the ancestral haplotype in Australian hemochromatosis patients may be associated with a common mutation in the gene. Am J Hum Genet 1995; 57:362-367.

10. Yaouanq J, Perichon M, Chorney M, Pontarotti P, Le Treut A, El Kahloun A, Mauvieux V, et al. Anonymous marker loci within $400 \mathrm{~kb}$ of HLA:A generate haplotypes in linkage disequilibrium with the hemochromatosis gene. Am J Hum Genet 1994;54:252-263.

11. Simon M, Le Mignon L, Fauchet R, Yaouanq J, David V, Edan G, Bourel M. A study of 609 haplotypes marking for the hemochromatosis gene: (1) mapping of the gene near the HLA-A locus and characters required to define a heterozygous population and (2) hypothesis concerning the underlying ause of hemochromatosis-HLA association. Am J Hum Genet 1987; 41:89-105

12. Olynik J, O'Neill R, Britton R, Bacon B. Determination of hepatic iron concentration in fresh and paraffin-embedded tissue: diagnostic implications. Gastroenterology 1994; 106:674-677.

13. Genethon. The Genethon Microsatellites Map Catalogue. Genethon, Human Genome Research Centre, Evry, France, 1992.

14. Weber J, Kwitek A, May P, Zoghbi H. Dinucleotide repeat polymorphism at the D6S105 locus. Nucleic Acids Res 1991;19:968.

15. Camaschella C, Roetto A, DeSandre G, Piperno A, Totaro A, Dianzani I, Gasparini P. Construction of a genetic map telomeric to HLA-A by microsatellite analysis. Mol Cell Probes 1993; 7:411-414.

16. Campbell R, Trowsdale J. Map of the human MHC. Immunol Today 1993; 14:349-352.

17. Mandelli C, Cesarini L, Piperno A, Fargion S, Fracanzani A, Barisani D, Conte D. Saturability of hepatic iron deposits in genetic hemochromatosis. Hepatology 1992; 16:956-9.

18. Piperno A, Fargion S, Panajotopoulos N, Del Ninno E, Fiorelli G. Idiopathic hemochromatosis and HLA antigens in Italy: is A3-Bw35 haplotype a marker for idiopathic hemochromatosis in north-east region? J Clin Pathol $1986 ; 39: 125-128$ 